OPEN ACCESS

Edited by:

Fabio Bagnoli,

GlaxoSmithKline, Italy

Reviewed by:

Mustafa Akkoyunlu,

United States Food and Drug

Administration, United States

Javier Carbone,

Gregorio Marañón Hospital, Spain

*Correspondence:

Maxime Hentzien

mhentzien@chu-reims.fr

Specialty section:

This article was submitted to Vaccines and Molecular Therapeutics,

a section of the journal

Frontiers in Immunology

Received: 08 October 2021 Accepted: 29 November 2021 Published: 20 December 2021

Citation:

Romaru J, Bahuaud M, Lejeune G, Hentzien M, Berger J-L, Robbins A, Lebrun D, N'Guyen Y, Bani-Sadr F, Batteux F and Servettaz A (2021) Single-Dose 13-Valent Conjugate Pneumococcal Vaccine in People Living With HIV - Immunological

Response and Protection.

Front. Immunol. 12:791147. doi: 10.3389/fimmu.2021.791147

\section{Single-Dose 13-Valent Conjugate Pneumococcal Vaccine in People Living With HIV - Immunological Response and Protection}

\author{
Juliette Romaru ${ }^{1}$, Mathilde Bahuaud ${ }^{2}$, Gauthier Lejeune ${ }^{1,3}$, Maxime Hentzien ${ }^{1 *}$, \\ Jean-Luc Berger ${ }^{1}$, Ailsa Robbins ${ }^{1,4}$, Delphine Lebrun ${ }^{3}$, Yohan N'Guyen ${ }^{1}$, \\ Firouzé Bani-Sadr ${ }^{1}$, Frédéric Batteux ${ }^{2}$ and Amélie Servettaz ${ }^{1,4}$ \\ 1 Department of Internal Medicine, Clinical Immunology and Infectious Diseases, Reims University Hospital, Reims, France, \\ 2 Plateforme d'Immunomonitoring Vaccinal, Laboratory of Immunology, Cochin Hospital and University Paris-Descartes, \\ APHP, Paris, France, ${ }^{3}$ Department of Internal Medicine and Infectious Diseases, $\mathrm{CH}$ de Charleville-Mézières, Charleville- \\ Mézières, France, ${ }^{4}$ Laboratory of Immunology, EA7509 IRMAIC, University of Reims Champagne-Ardenne (URCA), \\ Reims, France
}

Background: Patients living with HIV (PLHIV) are prone to invasive pneumococcal disease. The 13-valent conjugated pneumococcal vaccine (PCV13) is currently recommended for all PLHIV, followed in most guidelines by a 23-valent polysaccharide pneumococcal vaccine. Data are scarce concerning the immunological efficacy of PCV13 among PLHIV.

Objective: To assess the immunological response at one month, and the immunological protection at 1-, 6-, and 12 months in PLHIV with a CD4 cell count above 200 cells/ $\mu$ lafter a single dose of PCV13, as measured by both ELISA and opsonophagocytic assay (OPA).

Methods: PLHIV with CD4 cell count $>200$ cells/ul were included. Specific IgG serum concentrations for eight serotypes by ELISA and seven serotypes by OPA were measured at baseline, 1-, 6-, and 12 months after the PCV13 vaccination. Global response was defined as a two-fold increase from baseline of specific lgG antibody levels $(\mu \mathrm{g} / \mathrm{ml})$ assayed by ELISA or as a four-fold increase in OPA titer from baseline, for at least five serotypes targeted by PCV13. Global protection was defined as an IgG-concentration $\geq 1$ $\mu \mathrm{g} / \mathrm{ml}$ by ELISA or as an opsonization titer $\geq$ LLOQ by OPA for at least five tested serotypes targeted by PCV13. Factors associated with global response and global protection were assessed using logistic regression.

Results: Of the 38 PLHIV included, 57.9\% and 63.2\% were global responders, 92.1\% and $78.9 \%$ were globally protected at one month, and $64.7 \%$ and $55.9 \%$ were still protected at 12 months, by ELISA and OPA respectively. A CD4/CD8 ratio of $>0.8$ was significantly associated with a better global response by OPA (OR=6.11, $p=0.02)$, and a CD4 nadir <200 was significantly associated with a poorer global response by ELISA (OR=0.22, $\mathrm{p}=0.04$ ). A CD4 cell count nadir $<200$ and age over 50 years were associated with poorer global protection by OPA at $\mathrm{M} 1(\mathrm{OR}=0.18, \mathrm{p}=0.04)$ and $\mathrm{M} 12(\mathrm{OR}=0.15$, 
$\mathrm{p}=0.02)$, respectively. Plasma HIV RNA viral load $<40$ copies $/ \mathrm{ml}$ was significantly associated with a better global protection at M1 by ELISA and OPA (OR=21.33, $\mathrm{p}=0.025$ and $\mathrm{OR}=8.40, \mathrm{p}=0.04$ )

Conclusion: Vaccination with PCV13 in these patients induced immunological response and protection at one month. At one year, more than half of patients were still immunologically protected.

Keywords: HIV infection, pneumococcal conjugate vaccine (PCV), immunogenicity, immunological protection, immunological response, France, cohort study

\section{INTRODUCTION}

Streptococcus pneumoniae, formerly known as pneumococcus, causes severe infections such as bacterial pneumonia and invasive pneumococcal diseases (IPD) including meningitis, arthritis, and bacteremia. They are responsible for a high morbidity and mortality burden. S.pneumoniae possess a polysaccharide (PS) pneumococcal capsule which partly explains its virulence. More than 90 different serotypes have been distinguished (1) on the basis of the capsular antigen.

People living with HIV (PLHIV) are at higher risk of invasive pneumococcal diseases (IPD) than the general population. Despite the significant risk reduction conferred by combination antiretroviral therapy (cART) (2), PLHIV are still more susceptible to IPD and have a higher risk of recurrent pneumococcal infection in the year afterward (3-5). Factors likely to explain these differences are loss of memory cells, persistent inflammation, and nasopharyngeal pneumococcal carriage $(5,6)$.

To prevent pneumococcal disease, most guidelines, including those in France, recommend pneumococcal vaccines in PLHIV (7-9). In Europe, two vaccine formulations are currently available: the 23-valent pneumococcal polysaccharide vaccine (PPV23) and the 13-valent pneumococcal conjugate vaccine (PCV13). The PPV23 leads to a T-independent response, which limits the magnitude of the response and duration. The PCV13 elicits a T-cell dependent immune response $(10,11)$, characterized by improved antibody response, induction of memory cells, and increased response duration (11).

Two techniques can be used to measure immunological response: enzyme-linked immunosorbent assay (ELISA) and opsonophagocytic assay (OPA) $(12,13)$. ELISA only measures antibody levels whereas the OPA can measure the functional immunogenicity of the specific serotype antibodies produced and thereby likely reflects the in-vivo response more accurately (13). The OPA may therefore be a better surrogate marker of immune protection in immunocompromised patients than the ELISA which may be measuring nonfunctional antibodies (13-15).

An initial PCV13 is currently recommended for all PLHIV, followed in most guidelines by a PPV23 vaccine at least 2 months later to broaden serotype coverage $(7-9,16)$. The clinical efficacy of this combined vaccination schedule in PLHIV is unknown (17).

The optimal timing for the initial PCV13 vaccine remains unclear, but preferentially when there is a CD4 cell count above
200 cells/ $\mu$ and an undetectable viral load to strengthen immunological response.

Data are scarce concerning the immunological response to PCV13 vaccine among PLHIV, leading to a low overall pneumococcal vaccine coverage in this population (17).

The objective of the present study was therefore to assess the immunological response at one month and immunological protection at 1-, 6-, and 12 months after PCV13 administration, using both ELISA and OPA assays in a French cohort of PLHIV with a CD4 cell count above 200 cells/ $\mu$ l.

\section{MATERIALS AND METHODS}

\section{Study Design}

This prospective cohort study was conducted at the Reims University Hospital from December 2013 to July 2016.

\section{Study Population}

HIV1-infected subjects aged $\geq 18$ years old with a CD4 T-cell count $>200 \mathrm{cell} / \mu \mathrm{l}$, who had no history of anti-pneumococcal conjugate vaccination, had not received any pneumococcal polysaccharide vaccine in the last 3 years, and agreed to participate in the present study were included the day of their PCV13 vaccination. This was an exploratory observational study and recruitment was done using a pragmatic approach: all individuals fulfilling vaccination criteria and consenting to routine vaccination according to French guidelines were eligible and asked to participate in the study. The appropriate sample size could not be calculated beforehand due to the absence of previously published data for global response and protection definitions with ELISA and OPA with PCV13.

\section{Data Collection at Baseline and During Follow-Up}

At inclusion, a blood sample was collected for all patients before vaccination (M0), then a single $0.5 \mathrm{ml}$ intramuscular injection of PCV13 (Prevenar $13^{\circledR}$; Pfizer) was administered according to French guidelines.

The patients continued routine HIV care and were followed for up to one year without injection of further pneumococcal polysaccharide vaccine. Blood samples for immunological assessment were collected at baseline (M0) and during followup at 1- (M1), 6- (M6), and 12 months (M12). 
Sociodemographic characteristics, clinical data, and blood test results were collected at baseline using a dedicated case report form. HIV viral load and CD4 cell count were collected at baseline, and then at 1-, 6-, and 12- months when available.

\section{Immunogenicity Assessment}

All immunogenicity analyses were performed at the French referral laboratory for anti-pneumococcal serology and OPA (Cochin Hospital Center, Paris).

\section{ELISA}

IgG antibodies concentrations for eight pneumococcal serotypes $(4,6 \mathrm{~B}, 9 \mathrm{~V}, 14,18 \mathrm{C}, 7 \mathrm{~F}, 19 \mathrm{~F}$, and 23F) targeted by both PCV13 and PPSV23 and two targeted only by PPSV23 (10F and 15B) were determined using modified ELISA as previously described (18-21). Plates (Corning, Inc., Corning, NY) were coated with a serotype-specific pneumococcal PS antigen (American Type Culture Collection, Manassas, VA). Reference sera (007sp), control sera, or patient specimens were pre-absorbed with 5 $\mu \mathrm{g} / \mathrm{ml}$ pneumococcal C-polysaccharide (Statens Serum Institut, Copenhagen, Denmark) and $10 \mu \mathrm{g} / \mathrm{ml}$ serotype 22F capsular polysaccharide (American Type Culture Collection). Antipneumococcal antibody levels were determined in each specimen by analysis of linear regression plots compared with the reference serum (007sp) (National Institute for Biological Standards and Control) (NIBSC) (21).

\section{OPA}

OPA was performed for seven specific serotypes $(4,6 \mathrm{~B}, 9 \mathrm{~V}, 14$, $18 \mathrm{C}, 19 \mathrm{~F}$, and $23 \mathrm{~F})$ as previously described $(18,21,22)$ to determine functional antibody responses and measured by a multiplexed opsonophagocytic killing assay (MOPA). A detailed protocol is available online at www.vaccine.uab.edu. All serum samples were incubated at $56^{\circ} \mathrm{C}$ for $30 \mathrm{~min}$ before being tested. Sera were serially diluted in round-bottom 96-well plates (Corning Inc., Corning, NY). Frozen aliquots of target pneumococci were thawed, washed twice, diluted to a bacterial density of $\sim 50,000 \mathrm{CFU} / \mathrm{ml}$, and added to the plates. After 30 min of incubation at room temperature with shaking at $700 \mathrm{rpm}$, complement and HL60 cells (ATCC) that had been differentiated to phagocytes were added to each well. Plates were incubated in a tissue culture incubator $\left(37^{\circ} \mathrm{C}, 5 \% \mathrm{CO} 2\right)$ with shaking at 700 rpm. After a 45-min incubation, plates were placed on ice for 20 min. Ten $\mu$ l of each well were spotted onto four different ToddHewitt broth-yeast extract agar plates. After application of an overlay agar containing one of four antibiotics to each agar plate and overnight incubation at $37^{\circ} \mathrm{C}$, the number of bacterial colonies in the agar plates was enumerated. Opsonization titers (OT) were defined as interpolated reciprocal serum dilution that killed $50 \%$ of the bacteria in the assay. The assay sensitivity is the lowest dilution of sera tested (limit of detection: LOD), which is normally 4 for undiluted sera, and is the same for each serotype. However, to quantify functional antibodies with more precision, the lower limit of quantification (LLOQ) was determined for each serotype-specific assay during assay validation. The LLOQs for each serotype were: serotype 4: 24 , serotype $6 \mathrm{~B}$ : 132 , serotype $9 \mathrm{~V}$ : 39 , serotype $14: 85$, serotype $18 \mathrm{C}$ : 47 , serotype $19 \mathrm{~F}: 74$ and serotype 23F: 30. Titers higher than the LLOQs were considered accurate, and their values were reported. Titers below the LLOQs were set to a value of 2 (half a LOD) $(18,21,23)$. Before the OPA, all samples were tested on agar for their natural bactericidal effect. A drop of serum was placed on a Todd Hewitt agar onto which we also deposited a suspension of living pneumococcus. After one night at $37^{\circ} \mathrm{C}$, plates were examined for inhibition of colony growth indicating the presence of bactericidal agents in the serum. In case of bactericidal effect, the serum was not tested in OPA.

\section{Definitions of Serotype and Global Response and Protection}

The definitions used in our study were based on previously proposed criteria for response to pneumococcal vaccine among immunocompromised patients $(5,18,20,21,24)$ :

- Serotype specific IgG response for each serotype was defined as a two-fold increase from baseline of serotype specific IgG antibody levels $(\mu \mathrm{g} / \mathrm{ml})$ assayed by ELISA.

-OPA response for each serotype was defined as a four-fold increase in OPA titer from baseline

-Global responder to the vaccine by ELISA or OPA was defined as a patient who developed a response for at least five of the tested serotypes targeted by PCV13

-Protection was defined as an IgG-concentration $\geq 1 \mu \mathrm{g} / \mathrm{ml}$ by ELISA or as at least an opsonization titer $\geq$ LLOQ by OPA for the corresponding serotype.

-Global protection by ELISA or OPA was defined by a protection for at least five of the tested serotypes targeted by PCV13

\section{Statistical Analysis}

Qualitative values were reported as number and percentage, and compared with chi square test, or with Fisher's exact test, as appropriate. Quantitative values were reported as mean and standard deviation (SD), and compared using the Mann-Whitney $\mathrm{U}$ test. Serotype-specific IgG levels $(\mu \mathrm{g} / \mathrm{ml})$ and OPA titers were represented as geometric mean concentrations (GMC) or titers (GMT) with their corresponding 95\% confidence interval (CIs), respectively, at each time point. Correlation between values obtained by ELISA and OPA at each time point for each serotype were assessed using Spearman's Rho. The analysis of factors associated with global response at $\mathrm{M} 1$ and with global protection at M0, M6, and M12 was performed using univariable logistic regression. Quantitative explicative covariates were dichotomized at the median for age, CD4 and CD8 cell count, and neutrophil count (25). Due to the insufficient number of subjects included, no multivariable logistic regression was performed. A p-value below 0.05 was considered statistically significant. All statistical analyses were performed using SAS version 9.4 (SAS Institute Inc., Cary, North Carolina, USA.

\section{Ethics}

We received written and informed consent from every patient. This study was approved by the institutional review board of the Reims University Hospital in January 2015 and was conducted in accordance with the principles of the Declaration of Helsinki. 


\section{RESULTS}

\section{Baseline Demographics}

A total of 38 patients were included. Their mean age was $49.1 \pm$ 10.8 years, and $35(92.1 \%)$ of them were male. Baseline characteristics are detailed in Table 1. All patients but one were treated with combined antiretroviral therapy (cART) at the time of the PCV13 vaccination. Viral load $<40$ copies/ml was present in $86.8 \%$ of patients and the mean CD4 cell count was

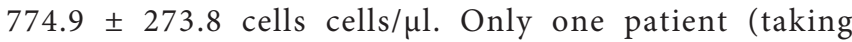
cotrimoxazole prophylaxis) received antibiotics at baseline, and no sera induced bactericidal effect in vitro before OPA. Mean

TABLE 1 | Baseline characteristics of 38 HIV-infected subjects with CD4 counts $\geq 200$ cells/ $\mu$ l vaccinated with 13-valent pneumococcal conjugate vaccine (PCV13).

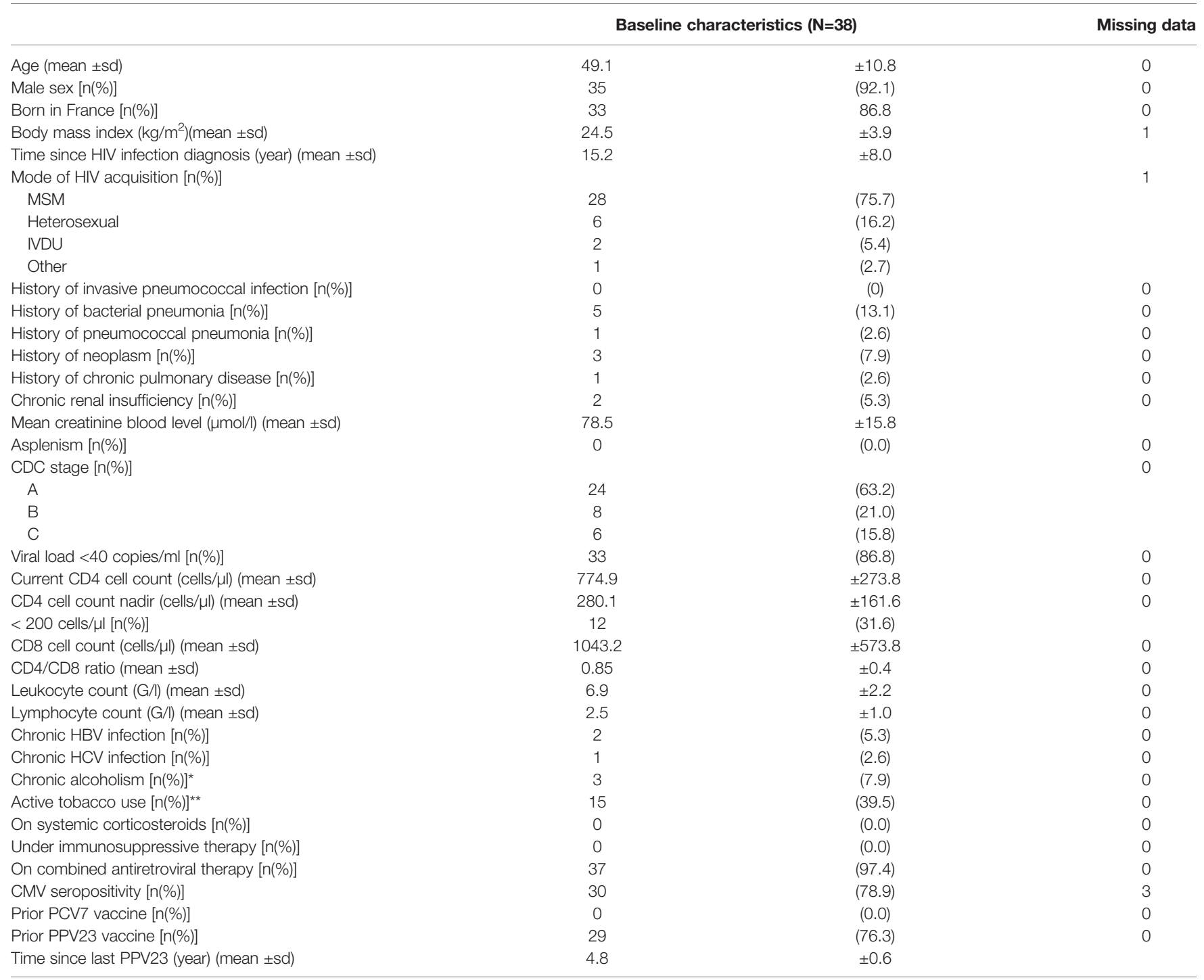

Sd, standard deviation;

MSM, Men who have sex with men;

IVDU, Intravenous Drug User:

$C D C$, Centers for disease control;

$H B V$, hepatitis B infection;

$H C V$, hepatitis $C$ infection;

NRTI, Nucleoside reverse transcriptase inhibitors;

NNRTI, Non-nucleoside reverse transcriptase inhibitors;

PI, Protease inhibitor;

IIN , Integrase inhibitors;

PCV7, 7-valent pneumococcal conjugate vaccine;

PPV23, 23-valent pneumococcal polysaccharide vaccine.

${ }^{*}$ Chronic alcoholism: more than $20 \mathrm{~g}$ of pure alcohol per day.

${ }^{*}$ Active tobacco use: active smokers in the last year. 
duration since HIV diagnosis was $15.2 \pm 8.0$ years. No patient had previously received the PCV7 or PCV13 vaccine, whereas 29 patients $(76.3 \%)$ had previously received the PPV23 vaccine with a mean time since the last PPV23 injection of $4.8 \pm 0.6$ years.

\section{Immunological Status at Baseline Toward Selected Pneumococcal Serotypes by ELISA and OPA in PLHIV}

GMC and GMT at baseline are presented in Table 2 and in Figures 1A, B. Protection rates at baseline, globally and by serotype, by ELISA and OPA, are presented in Table 6.

At baseline, IgG antibodies GMCs were higher than the $1 \mu \mathrm{g} /$ $\mathrm{ml}$ threshold for all serotypes except serotypes $4,9 \mathrm{~V}$, and $18 \mathrm{C}$. By contrast none of the GMTs for the seven serotypes measured by OPA were above their respective LLOQ (Table 2).

Baseline serotype protection in ELISA ranged from $21.0 \%$ to $86.8 \%$ of patients according to the concerned serotype. Baseline serotype protection by OPA ranged from $21.0 \%$ to $39.5 \%$ of patients according to the concerned serotype. Half of our population was considered already globally protected according to ELISA and about $15 \%$ according to OPA before PCV13 vaccination (Table 6).

Patients with a CD4 cell count over the population median of 750 cells/ $\mu$ were significantly more likely to be globally protected at baseline, when tested by ELISA $(\mathrm{OR}=4.80$, $\mathrm{p}=0.03$ ) than other PLHIV (Table 3). No factor was significantly associated with global protection by OPA at baseline.

\section{One-Month Immunological Response After PCV13, Assessed by ELISA and OPA, in PLHIV}

At one-month post-vaccination, we observed a 2.4- to 3.5-fold increase in GMCs compared to baseline for all serotypes contained in PCV13, and no increase in the serotypes 10A and 15B which are not present in PCV13 (Table 2). We found similar results by OPA, as GMTs increased 9.3-21.7-fold for all serotypes (Table 2). The one-month GMCs ranged from 1.3 to $10.6 \mu \mathrm{g} / \mathrm{ml}$ and the one-month GMTs ranged from 169.9 to 473.7 .

According to the definition of immunological response for a serotype, the response rates ranged from $42.1 \%$ to $71.0 \%$ by ELISA and from 57.9 to $76.3 \%$ by OPA. Twenty-two (57.9\%) and $24(63.2 \%)$ subjects were considered to be global responders by ELISA and by OPA, respectively (Table 4).

Factors associated with a one-month global response, by ELISA and OPA, are presented in Table 5. By ELISA, having had a CD4 nadir below 200 was significantly associated with a lower risk of developing a global one-month response $(\mathrm{OR}=0.22$, $\mathrm{p}=0.04$ ). By OPA, a CD4/CD8 ratio $>0.8$ was significantly associated with a better chance of obtaining a global onemonth immunological response $(\mathrm{OR}=6.11, \mathrm{p}=0.019)$.

TABLE 2 | Geometric mean concentrations (GMC) and geometric mean titers (GMT) to pneumococcal serotypes before and at one, six and twelve months after administration of 13-valent pneumococcal conjugate vaccine (PCV13) in 38 HIV-infected subjects with CD4 cell count $\geq 200$ cells/ $/$ l.

\begin{tabular}{|c|c|c|c|c|c|c|c|c|c|c|c|c|c|c|c|c|}
\hline \multirow[t]{3}{*}{ Serotype } & \multicolumn{8}{|c|}{ ELISA GMC } & \multicolumn{8}{|c|}{ OPA GMT } \\
\hline & \multicolumn{2}{|c|}{ MO } & \multicolumn{2}{|c|}{ M1 } & \multicolumn{2}{|c|}{ M6 } & \multicolumn{2}{|c|}{ M12 } & \multicolumn{2}{|c|}{ MO } & \multicolumn{2}{|c|}{ M1 } & \multicolumn{2}{|c|}{ M6 } & \multicolumn{2}{|c|}{ M12 } \\
\hline & $\begin{array}{c}\text { GMC } \\
(\mu \mathrm{g} / \mathrm{mL})\end{array}$ & $\begin{array}{c}(95 \% \\
\mathrm{Cl})\end{array}$ & $\begin{array}{c}\text { GMC } \\
(\mu \mathrm{g} / \mathrm{mL})\end{array}$ & $\begin{array}{c}(95 \% \\
\mathrm{Cl})\end{array}$ & $\begin{array}{c}\text { GMC } \\
(\mu \mathrm{g} / \mathrm{mL})\end{array}$ & $\begin{array}{c}(95 \% \\
\mathrm{Cl})\end{array}$ & $\begin{array}{c}\text { GMC } \\
(\mu \mathrm{g} / \mathrm{mL})\end{array}$ & $\begin{array}{c}(95 \% \\
\mathrm{Cl})\end{array}$ & GMT & $\begin{array}{c}(95 \% \\
\mathrm{Cl})\end{array}$ & GMT & $(95 \% \mathrm{Cl})$ & GMT & $\begin{array}{c}(95 \% \\
\mathrm{Cl})\end{array}$ & GMT & $\begin{array}{c}(95 \% \\
\mathrm{Cl})\end{array}$ \\
\hline 4 & 0.6 & $\begin{array}{c}(0.4- \\
0.7)\end{array}$ & 1.9 & $\begin{array}{c}(1.4- \\
2.8)\end{array}$ & 1.0 & $\begin{array}{c}(0.8- \\
1.4)\end{array}$ & 0.8 & $\begin{array}{c}(0.6- \\
1.1)\end{array}$ & 8.3 & $\begin{array}{l}(4.3- \\
15.9)\end{array}$ & 169.9 & $\begin{array}{l}(80.6- \\
357.8)\end{array}$ & 37.6 & $\begin{array}{c}(15.8- \\
89.3)\end{array}$ & 36.3 & $\begin{array}{l}(16.9- \\
77.9)\end{array}$ \\
\hline $6 B$ & 1.7 & $\begin{array}{l}(1.2- \\
2.4)\end{array}$ & 4.1 & $\begin{array}{l}(2.7- \\
6.2)\end{array}$ & 2.8 & $\begin{array}{l}(1.8- \\
4.2)\end{array}$ & 2.5 & $\begin{array}{l}(1.7- \\
3.8)\end{array}$ & 18.1 & $\begin{array}{l}(9.1- \\
35.8)\end{array}$ & 390.7 & $\begin{array}{c}(186.3- \\
819.5)\end{array}$ & 171.7 & $\begin{array}{l}(83.6- \\
352.5)\end{array}$ & 111.6 & $\begin{array}{l}(54.9- \\
226.9)\end{array}$ \\
\hline $9 \mathrm{~V}$ & 1.0 & $\begin{array}{c}(0.6- \\
1.6)\end{array}$ & 2.3 & $\begin{array}{c}(1.5- \\
3.6)\end{array}$ & 1.5 & $\begin{array}{l}(1.0- \\
2.2)\end{array}$ & 1.3 & $\begin{array}{c}(0.8- \\
2.0)\end{array}$ & 13.0 & $\begin{array}{l}(7.3- \\
23.1)\end{array}$ & 183.6 & $\begin{array}{l}(94.8- \\
355.7)\end{array}$ & 81.2 & $\begin{array}{l}(37.6- \\
175.0)\end{array}$ & 51.9 & $\begin{array}{l}(23.9- \\
112.7)\end{array}$ \\
\hline 14 & 4.2 & $\begin{array}{l}(2.5- \\
7.0)\end{array}$ & 10.6 & $\begin{array}{l}(6.6- \\
17.0\end{array}$ & 7.6 & $\begin{array}{l}(4.7- \\
12.3)\end{array}$ & 7.6 & $\begin{array}{l}(4.9- \\
12.0)\end{array}$ & 42.8 & $\begin{array}{l}(17.0- \\
107.9)\end{array}$ & 473.7 & $\begin{array}{l}(206.1- \\
1089.1)\end{array}$ & 241.7 & $\begin{array}{l}(97.9- \\
596.3)\end{array}$ & 223.4 & $\begin{array}{l}(98.8- \\
505.4)\end{array}$ \\
\hline $18 \mathrm{C}$ & 0.9 & $\begin{array}{c}(0.6- \\
1.3)\end{array}$ & 3.0 & $\begin{array}{l}(2.1- \\
4.3)\end{array}$ & 1.7 & $\begin{array}{l}(1.2- \\
2.4)\end{array}$ & 1.6 & $\begin{array}{l}(1.1- \\
2.3)\end{array}$ & 19.6 & $\begin{array}{l}(9.8- \\
39.7)\end{array}$ & 309.1 & $\begin{array}{c}(163.8- \\
583.3)\end{array}$ & 120.6 & $\begin{array}{l}(57.0)- \\
255.5)\end{array}$ & 89.3 & $\begin{array}{l}(43.8- \\
182.2)\end{array}$ \\
\hline $19 F$ & 2.8 & $\begin{array}{l}(2.0- \\
3.9)\end{array}$ & 6.6 & $\begin{array}{c}(4.8- \\
9.0)\end{array}$ & 3.7 & $\begin{array}{l}(2.6- \\
5.2)\end{array}$ & 2.9 & $\begin{array}{l}(1.9- \\
4.3)\end{array}$ & 31.1 & $\begin{array}{l}(14.8- \\
65.7)\end{array}$ & 287.9 & $\begin{array}{c}(144.2- \\
574.7)\end{array}$ & 130.7 & $\begin{array}{l}(60.9- \\
280.7)\end{array}$ & 124.9 & $\begin{array}{l}(66.2- \\
235.3)\end{array}$ \\
\hline $23 F$ & 1.3 & $\begin{array}{c}(0.9- \\
1.9)\end{array}$ & 3.6 & $\begin{array}{c}(2.3- \\
5.7)\end{array}$ & 2.4 & $\begin{array}{l}(1.4- \\
4.0)\end{array}$ & 1.6 & $\begin{array}{l}(0.9- \\
2.8)\end{array}$ & 14.0 & $\begin{array}{l}(6.1- \\
31.8)\end{array}$ & 222.9 & $\begin{array}{l}(80.3- \\
618.4)\end{array}$ & 90.7 & $\begin{array}{l}(28.8- \\
286.0)\end{array}$ & 79.4 & $\begin{array}{l}(28.2- \\
223.6)\end{array}$ \\
\hline $7 F$ & 1.1 & $\begin{array}{c}(0.7- \\
1.9)\end{array}$ & 3.8 & $\begin{array}{c}(2.4- \\
6.1)\end{array}$ & 2.6 & $\begin{array}{l}(1.7- \\
4.1)\end{array}$ & 1.8 & $\begin{array}{l}(1.2- \\
2.9)\end{array}$ & & & & & & & & \\
\hline $10 A^{*}$ & 1.3 & $\begin{array}{l}(0.7- \\
2.3)\end{array}$ & 1.3 & $\begin{array}{l}(0.7- \\
2.3)\end{array}$ & 1.2 & $\begin{array}{l}(0.7- \\
2.3)\end{array}$ & 1.1 & $\begin{array}{c}(0.6- \\
2.0)\end{array}$ & & & & & & & & \\
\hline $15 B^{\star}$ & 1.8 & $\begin{array}{l}(1.0- \\
3.2)\end{array}$ & 2.2 & $\begin{array}{c}(1.3- \\
3.7)\end{array}$ & 1.8 & $\begin{array}{l}(1.0- \\
3.1)\end{array}$ & 1.8 & $\begin{array}{c}(1.1- \\
3.0)\end{array}$ & & & & & & & & \\
\hline
\end{tabular}

Cl, confidence intervals.

GMC, geometric mean concentrations; GMT, geometric mean titers.

*Targeted by PPV23 but not PCV13. 
One-Month, 6-Month, and 12-Month Immunological Protection After PCV13, Analyzed by ELISA and OPA, in PLHIV

At one month, the protection rate for each serotype included in PCV13 ranged from $71 \%$ to $97.4 \%$ by ELISA and from $76.3 \%$ to $86.8 \%$ by OPA (Table 6). Thirty-five $(92.1 \%)$ and thirty $(78.9 \%)$ patients were globally protected according to ELISA and OPA measures, respectively.

GMCs at 6 and 12 months remained higher than baseline for all serotypes contained in the PCV13 but decreased compared to
M1 (Figure 1A). Only serotype 4 had GMC $<1 \mu \mathrm{g} / \mathrm{ml}$ in ELISA at 12 months. By OPA, GMT also decreased but still exceeded the protective threshold for all serotypes at M12.

Consequently, at six months, the protection rate for each serotype included in PCV13 ranged from $44.4 \%$ to $91.7 \%$ by ELISA and $60 \%$ to $71.4 \%$ by OPA. Twenty-six $(72.2 \%)$ and twenty-one $(60.0 \%)$ patients were globally protected by ELISA and by OPA, respectively (Table 6).

At 12 months, the protection rate for each serotype included in PCV 13 ranged from $41.2 \%$ to $100.0 \%$ by ELISA and $61.8 \%$ to

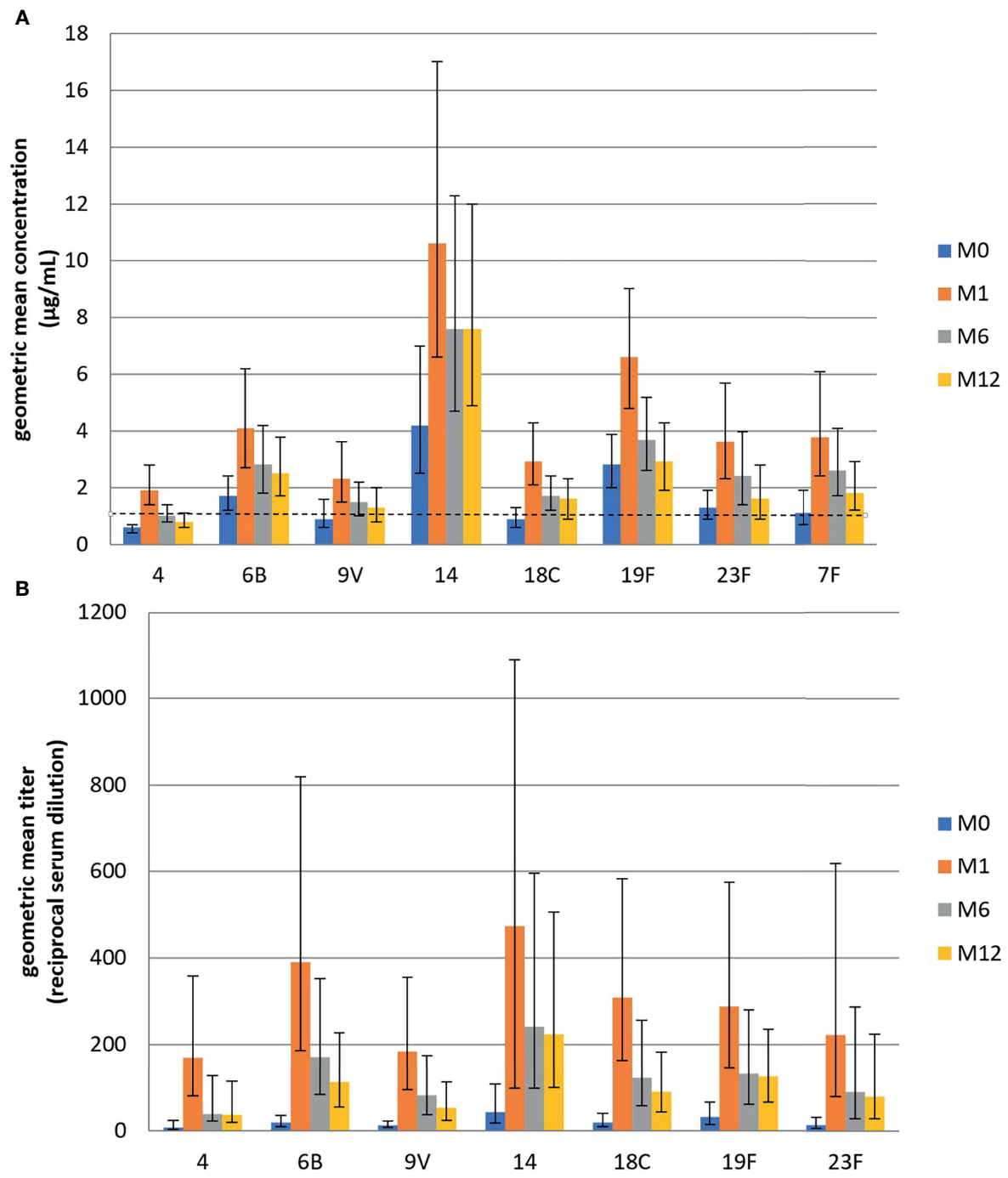

FIGURE 1 | (A) Geometric mean concentration (GMC) of IgG antibodies toward eight pneumococcal serotypes targeted by PCV13, measured by ELISA before and one, six, and twelve months after administration of 13-valent pneumococcal conjugate vaccine among 38 HIV-infected subjects with CD4 cell count $\geq 200$ cells/ $\mu$. (B) Geometric mean titers (GMT) of opsonophagocytic activity measured by OPA toward seven pneumococcal serotypes targeted by PCV13, before and one, six, and twelve months after administration of 13-valent pneumococcal conjugate vaccine at one, six, and twelve months among 38 HIV-infected subjects with CD4 cell count $\geq 200$ cells $/ \mu$ l. 
TABLE 3 | Factors associated with global protection at baseline by OPA and ELISA among 38 HIV-infected subjects with CD4 cell count $\geq 200$ cells/ $\mu$ l.

\begin{tabular}{|c|c|c|c|c|c|c|}
\hline Variable & \multicolumn{3}{|c|}{ ELISA } & \multicolumn{3}{|c|}{ OPA } \\
\hline CD4 cell count $>750$ cells $/ \mu l$ & 4.80 & $1.20-19.13$ & 0.03 & 2.27 & $0.36-14.18$ & 0.38 \\
\hline $\mathrm{CD} 4 / \mathrm{CD} 8$ ratio $>0.8$ & 0.43 & $0.12-1.59$ & 0.21 & 0.50 & $0.08-3.13$ & 0.46 \\
\hline Plasma HIV RNA viral load, $<40$ copies $/ \mathrm{ml}$ & 6.15 & $0.62-61.37$ & 0.12 & >999.99 & $<0.001->999.99$ & 0.97 \\
\hline Neutrophil count $>3500 \mathrm{cell} / \mu \mathrm{l}$ & 1.07 & $0.29-3.92$ & 0.92 & 9.54 & $0.99-92.2$ & 0.051 \\
\hline Body mass index $\left(\mathrm{kg} / \mathrm{m}^{2}\right)$ & 2.25 & $0.54-9.34$ & 0.26 & 2.10 & $0.36-12.31$ & 0.41 \\
\hline CMV seropositivity & 6.91 & $0.68-69.86$ & 0.10 & $>999.99$ & $<0.001->999.99$ & 0.97 \\
\hline Time since HIV infection diagnosis & 1.44 & $0.39-5.28$ & 0.58 & 4.41 & $0.46-42.13$ & 0.20 \\
\hline History of bacterial pneumonia & 1.25 & $0.18-8.50$ & 0.82 & 1.40 & $0.13-15.26$ & 0.78 \\
\hline Nadir CD4 cell count $<200$ cells/uL & 0.45 & $0.11-1.80$ & 0.26 & $<0.001$ & $<0.001->999.99$ & 0.95 \\
\hline Active tobacco use & 0.36 & $0.09-1.36$ & 0.13 & 0.73 & $0.12-4.59$ & 0.74 \\
\hline
\end{tabular}

PPV23, 23-valent pneumococcal polysaccharide vaccine Globally protected was defined as a patient who developed a protection for at least five of the tested serotypes targeted by PCV13.

TABLE 4 | One-month immunological response after administration of 13-valent pneumococcal conjugate vaccine (PCV13) among 38 HIV-infected subjects with CD4 cell count $\geq 200$ cells/ $\mu$ l.

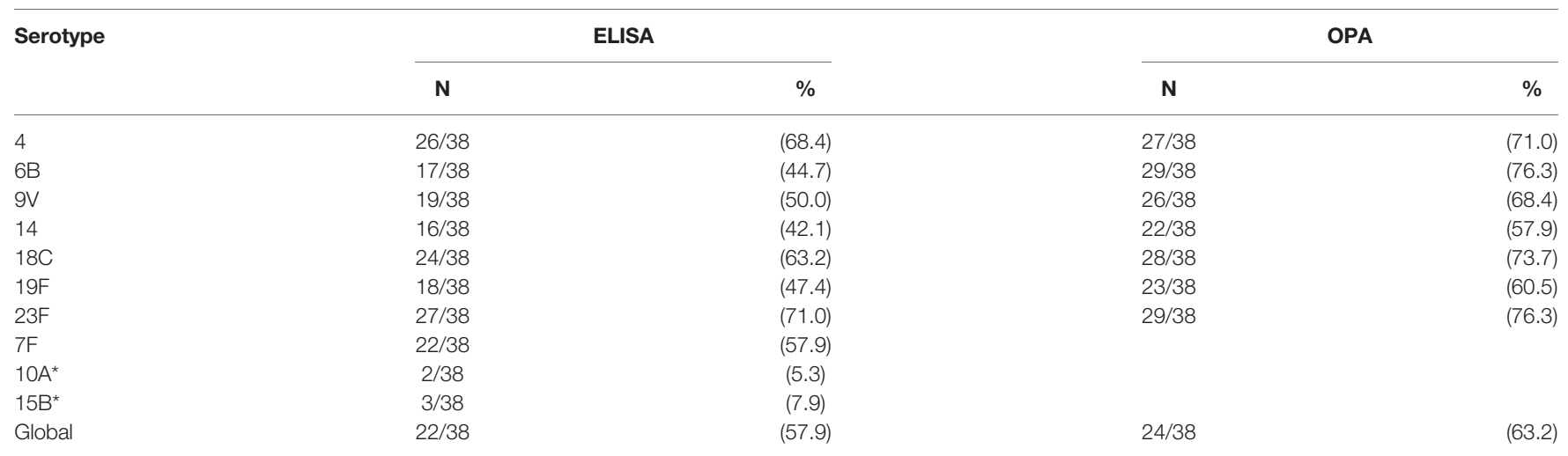

Responses was defined as a two-fold increase of serotype specific lgG antibody levels ( $\mu \mathrm{g} / \mathrm{ml})$ or as a four-fold increase in OPA titer from baseline.

Global response is defined as a patient who developed a response for at least five of the tested serotypes.

*The serotypes $10 \mathrm{~A}$ and 15B are targeted by PPV23 but not PCV13.

$70.6 \%$ by OPA. Twenty-two (64.7\%) and nineteen $(55.9 \%)$ patients were globally protected by ELISA and by OPA, respectively (Table 6).

No patient developed IPD during the study and follow-up after a mean follow-up of 6.8 years.

\section{Factors Associated With 1-Month and 12-Months Global Protection After PCV13, Analyzed by ELISA and OPA, in PLHIV}

The factors associated with one-month and twelve-month global protection, by ELISA and OPA, are presented in Tables 7 and 8, respectively.

Patients who were global responders at one month were not significantly more likely to obtain global immunological protection at 12 months whether assessed by ELISA or OPA ( $\mathrm{p}=0.28$ and $\mathrm{p}=0.27$, respectively).

One month after vaccination, PLHIV with a CD4 cell count nadir below 200 cells/ $\mu$ l were significantly less globally protected
$(\mathrm{OR}=0.18, \mathrm{p}=0.04)$ by OPA. PLHIV with a plasma HIV RNA viral load $<40$ copies/ml were significantly more frequently globally protected $(\mathrm{OR}=21.33, \mathrm{p}=0.025$ and $\mathrm{OR}=8.40, \mathrm{p}=0.04)$ by ELISA and OPA, respectively.

Twelve months after vaccination, patients aged over 50 were less likely to still be protected according to OPA results $(\mathrm{OR}=0.15, \mathrm{p}=0.02)$. We found no factors significantly associated with global protection at M12 by ELISA.

\section{Correlation Between ELISA and OPA Methods at Baseline, M1, M6, and M12, in PLHIV}

Given that two techniques were systematically used to assess anti-pneumococcal immunological response and protection in our population, we analyzed the correlation between the results obtained from both techniques.

There was a significant positive correlation between values obtained for all assessed serotypes by ELISA and OPA (moderate 
TABLE 5 | Factors associated with global response one month after administration of 13-valent pneumococcal conjugate vaccine, by OPA and ELISA, among 38 HIVinfected subjects with CD4 cell count $\geq 200$ cells $/ \mu$ l.

\begin{tabular}{|c|c|c|c|c|c|c|}
\hline \multirow[t]{2}{*}{ Variable } & \multicolumn{3}{|c|}{ ELISA } & \multicolumn{3}{|c|}{ OPA } \\
\hline & Odds ratio & Confidence interval & $\mathbf{p}$ & Odds ratio & Confidence interval & $\mathbf{p}$ \\
\hline CD4 cell count $>750$ cells $/ \mu l$ & 0.65 & $0.18-2.37$ & 0.51 & 1.58 & $0.42-5.95$ & 0.50 \\
\hline CD8 cell count > 800 cells $/ \mu l$ & 1.4 & $0.39-5.28$ & 0.58 & 0.87 & $0.25-3.35$ & 0.86 \\
\hline CD4/CD8 ratio $>0.8$ & 2.0 & $0.54-7.45$ & 0.30 & 6.11 & $1.34-27.96$ & 0.02 \\
\hline Plasma HIV RNA viral load, <40 copies/ml & 0.30 & $0.03-2.98$ & 0.30 & 0.38 & $0.039-3.84$ & 0.41 \\
\hline Neutrophil count $>3500 \mathrm{cell} / \mu \mathrm{l}$ & 0.89 & $0.24-3.28$ & 0.86 & 0.60 & $0.16-2.28$ & 0.45 \\
\hline CDC stage $\mathrm{C}$ & 0.68 & $0.12-3.93$ & 0.67 & 1.2 & $0.19-7.57$ & 0.85 \\
\hline Age $>50$ years & 0.31 & $0.08-1.22$ & 0.09 & 0.29 & $0.07-1.18$ & 0.08 \\
\hline Body mass index $\left(\mathrm{kg} / \mathrm{m}^{2}\right)$ & 2.25 & $0.54-9.34$ & 0.26 & 2.82 & $0.62-12.89$ & 0.18 \\
\hline CMV seropositivity & 2.25 & $0.33-15.54$ & 0.41 & 3.00 & $0.43-20.94$ & 0.27 \\
\hline Time since HIV infection diagnosis & 0.45 & $0.12-1.75$ & 0.25 & 0.66 & $0.17-2.55$ & 0.54 \\
\hline History of bacterial pneumonia & 1.10 & $0.16-7.5$ & 0.92 & 2.60 & $0.26-25.92$ & 0.41 \\
\hline CD4 cell count nadir <200cells/ $\mu$ l & 0.22 & $0.05-0.96$ & 0.04 & 0.26 & $0.06-1.11$ & 0.07 \\
\hline Active tobacco use & 1.15 & $0.31-4.33$ & 0.83 & 0.80 & $0.21-3.06$ & 0.74 \\
\hline Prior PPV23 vaccine & 0.61 & $0.13-2.95$ & 0.54 & 1.52 & $0.33-6.96$ & 0.59 \\
\hline
\end{tabular}

PPV23, 23-valent pneumococcal polysaccharide vaccine.

Definition of a global responder: patient who developed a response for at least five of the tested serotypes targeted by PCV13.

Bold values indicate $p<0.05$.

TABLE 6 | Proportion of protected patients defined as patients who had a level $\geq 1 \mu \mathrm{g} / \mathrm{ml}$ of Streptococcus pneumoniae polysaccharide-specific lgG or as at least an opsonization titer $\geq$ LLOQ by OPA at baseline, one, six and twelve months after administration of 13-valent pneumococcal conjugate vaccine among 38 HIV-infected subjects with CD4 cell count $\geq 200$ cells/ $\mu$ l.

\begin{tabular}{|c|c|c|c|c|c|c|c|c|c|c|c|c|c|c|c|c|}
\hline \multirow[t]{3}{*}{ Serotype } & \multicolumn{8}{|c|}{ ELISA } & \multicolumn{8}{|c|}{ OPA } \\
\hline & \multicolumn{2}{|c|}{ Mo } & \multicolumn{2}{|c|}{ M1 } & \multicolumn{2}{|c|}{ M6 } & \multicolumn{2}{|c|}{ M12 } & \multicolumn{2}{|c|}{ MO } & \multicolumn{2}{|c|}{ M1 } & \multicolumn{2}{|c|}{ M6 } & \multicolumn{2}{|c|}{ M12 } \\
\hline & $\mathbf{N}$ & $\%$ & $\mathbf{N}$ & $\%$ & $\mathbf{N}$ & $\%$ & $\mathbf{N}$ & $\%$ & $\mathbf{N}$ & $\%$ & $\mathbf{N}$ & $\%$ & $\mathbf{N}$ & $\%$ & $\mathbf{N}$ & $\%$ \\
\hline 4 & $8 / 38$ & 21.0 & $27 / 38$ & 71.0 & $16 / 36$ & 44.4 & $14 / 34$ & 41.2 & $10 / 38$ & 26.3 & $32 / 38$ & 84.2 & $21 / 35$ & 60.0 & $22 / 34$ & 64.7 \\
\hline $6 \mathrm{~B}$ & $28 / 38$ & 73.7 & $34 / 38$ & 89.5 & $27 / 36$ & 75.0 & $26 / 34$ & 76.8 & $8 / 38$ & 21.0 & $31 / 38$ & 81.6 & $21 / 35$ & 60.0 & $21 / 34$ & 61.8 \\
\hline $9 \mathrm{~V}$ & $17 / 38$ & 44.7 & 2638 & 68.4 & $22 / 36$ & 61.1 & $16 / 34$ & 46.1 & $10 / 38$ & 26.3 & $31 / 38$ & 81.6 & $23 / 35$ & 65.7 & $21 / 34$ & 61.8 \\
\hline 14 & $32 / 38$ & 84.2 & $37 / 38$ & 97.4 & $34 / 36$ & 94.4 & $34 / 34$ & 100.0 & $15 / 38$ & 39.47 & $30 / 38$ & 78.9 & 23/35 & 65.7 & $24 / 34$ & 70.6 \\
\hline $18 \mathrm{C}$ & $20 / 38$ & 52.6 & $33 / 38$ & 86.8 & $26 / 36$ & 72.2 & $22 / 34$ & 64.7 & $14 / 38$ & 36.8 & $33 / 38$ & 86.8 & $25 / 35$ & 71.4 & $23 / 64$ & 67.6 \\
\hline $19 \mathrm{~F}$ & $33 / 38$ & 86.8 & $37 / 38$ & 97.4 & $33 / 36$ & 91.7 & $28 / 34$ & 82.3 & $14 / 38$ & 38.8 & $31 / 38$ & 81.6 & $22 / 35$ & 62.9 & $22 / 34$ & 64.7 \\
\hline $23 \mathrm{~F}$ & $21 / 38$ & 55.3 & $34 / 38$ & 89.5 & $28 / 36$ & 77.8 & $21 / 34$ & 61.8 & $9 / 38$ & 23.7 & $29 / 38$ & 76.3 & 23/35 & 65.7 & $22 / 34$ & 64.7 \\
\hline $7 F$ & $16 / 38$ & 42.1 & $30 / 38$ & 78.9 & $24 / 34$ & 70.6 & $24 / 34$ & 70.6 & & & & & & & & \\
\hline $10 A^{*}$ & $18 / 38$ & 47.4 & $18 / 38$ & 47.4 & $15 / 34$ & 44.1 & $16 / 34$ & 47.1 & & & & & & & & \\
\hline $15 \mathrm{~B}^{\star}$ & $25 / 38$ & 65.8 & $26 / 38$ & 68.4 & $21 / 34$ & 61.8 & $20 / 34$ & 58.8 & & & & & & & & \\
\hline Global & $21 / 38$ & 55.3 & $35 / 38$ & 92.1 & $26 / 36$ & 72.2 & $22 / 34$ & 64.7 & $6 / 38$ & 15.8 & $30 / 38$ & 78.9 & $21 / 35$ & 60.0 & $19 / 34$ & 55.9 \\
\hline
\end{tabular}

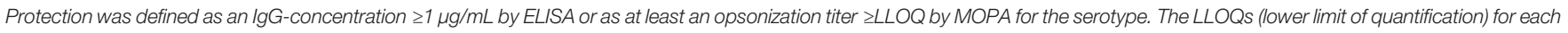
serotype were: serotype 4: 24, serotype 6B: 132, serotype 9V: 39, serotype 14: 85, serotype 18C: 47, serotype 19F: 74 and serotype $23 F: 30$.

Globally protected was defined as a patient who developed a protection for at least five of the tested serotypes targeted by PCV13.

${ }^{*}$ The serotypes $10 \mathrm{~A}$ and $15 \mathrm{~B}$ are targeted by PPV23 but not PCV13.

correlation for all serotypes at each timepoint except for serotype 14 which had a strong correlation, Table 9).

A strong correlation was found between global responses assessed by ELISA and OPA (Rho=0.67, $\mathrm{p}<0.0001$ ). Correlations were found between OPA and ELISA for immunological responses to serotypes $4(\mathrm{p}=0.0002), 9 \mathrm{~V}(\mathrm{p}=0.04), 14$ $(\mathrm{p}<0.0001), 18 \mathrm{C}(\mathrm{p}<0.0001)$, and 19F $(\mathrm{p}=0.005)$.

There was a weak correlation of borderline significance between ELISA and OPA for global protection at M12 (Rho=0.33, $\mathrm{p}=0.053$ ). For the serotype tested and methods, we found a significant serotype correlation between ELISA and OPA for only three serotypes [4 $(p=0.03), 19 F(p=0.006)$, and $23 \mathrm{~F}(\mathrm{p}=0.01)]$.

\section{DISCUSSION}

In our study, we evaluated the PCV13 immunological response and the global protection before and up to one year after a single dose of vaccination in PLHIV by combining two methods (ELISA and OPA). To the best of our knowledge, this study is the first to evaluate qualitatively (immunological response and protection) and quantitatively anti-pneumococcal humoral immunity after a single dose of PCV13 over a one-year period and its associated factors in PLHIV. These data may help clinicians improve PCV13 vaccine strategy and monitoring.

Although immunogenicity studies of PCV7 in HIV-infected adults have already been published (27-30), PCV13 vaccination- 
TABLE 7 | Factors associated with global protection one month after administration of 13-valent pneumococcal conjugate vaccine, by OPA and ELISA, among 38 HIVinfected subjects with CD4 cell count $\geq 200$ cells $/ \mu$ l.

\begin{tabular}{|c|c|c|c|c|c|c|}
\hline \multirow[t]{2}{*}{ Variable } & \multicolumn{3}{|c|}{ ELISA } & \multicolumn{3}{|c|}{ OPA } \\
\hline & Odds ratio & Confidence interval & $\mathbf{p}$ & Odds ratio & Confidence interval & $\mathbf{p}$ \\
\hline CD4 cell count > 750 cells/ $\mu$ (median) & $>999.99$ & $<0.001->999.99$ & 0.95 & 3.92 & $0.68-22.70$ & 0.13 \\
\hline CD8 cell count $>800$ cells $/ \mu l$ & 0.59 & $0.05-7.17$ & 0.68 & 0.69 & $0.14-3.40$ & 0.64 \\
\hline CD4/CD8 ratio $>0.8$ & >999.99 & $<0.001->999.99$ & 0.95 & 3.43 & $0.59-19.80$ & 0.17 \\
\hline Plasma HIV RNA viral load, <40 copies/ml & 21.33 & $1.47-310.01$ & 0.025 & 8.40 & $1.11-63.73$ & 0.04 \\
\hline Neutrophil count $>3500 \mathrm{cell} / \mu \mathrm{l}$ & 1.50 & $0.12-18.12$ & 0.75 & 2.62 & $0.45-15.16$ & 0.28 \\
\hline CDC stage C & 0.33 & $0.025-4.40$ & 0.40 & 0.46 & $0.07-3.13$ & 0.43 \\
\hline Age $>50$ years & 0.53 & 0.04-6.39 & 0.62 & 0.60 & $0.12-2.97$ & 0.53 \\
\hline Body mass index $\left(\mathrm{kg} / \mathrm{m}^{2}\right)$ & 1.09 & $0.09-13.30$ & 0.95 & 0.88 & $0.17-4.45$ & 0.87 \\
\hline CMV seropositivity & 3.5 & $0.25-48.03$ & 0.35 & 0.82 & $0.08-8.60$ & 0.87 \\
\hline Time since HIV infection diagnosis & 0.67 & $0.05-8.06$ & 0.75 & 0.78 & $0.16-3.90$ & 0.77 \\
\hline History of bacterial pneumonia & 0.26 & $0.02-3.53$ & 0.31 & 0.33 & $0.04-2.45$ & 0.28 \\
\hline Nadir CD4 count cell/ $\mu \mathrm{L}<200$ cells $/ \mu \mathrm{l}$ & 0.20 & $0.02-2.46$ & 0.21 & 0.18 & $0.03-0.96$ & 0.04 \\
\hline Active tobacco use & 1.33 & $0.11-16.14$ & 0.82 & 0.58 & $0.12-2.79$ & 0.50 \\
\hline Prior PPV23 vaccine & $<0.001$ & $<0.001->999.99$ & 0.95 & 1.09 & $0.18-6.69$ & 0.92 \\
\hline
\end{tabular}

PPV23, 23-valent pneumococcal polysaccharide vaccine.

Globally protected was defined as a patient who developed a protection for at least five of the tested serotypes targeted by PCV13.

Bold values indicate $p<0.05$.

TABLE 8 | Factors associated with global protection twelve months after administration of 13-valent pneumococcal conjugate vaccine, by Elisa and OPA, among 38 HIV-infected subjects with CD4 cell count $\geq 200$ cells/ $\mu$ l.

\begin{tabular}{|c|c|c|c|c|c|c|}
\hline \multirow[t]{2}{*}{ Variable } & \multicolumn{3}{|c|}{ ELISA } & \multicolumn{3}{|c|}{ OPA } \\
\hline & Odds ratio & Confidence interval & $\mathbf{p}$ & Odds ratio & Confidence interval & $\mathbf{p}$ \\
\hline CD4 cell count $>750$ cells $/ \mu l$ & 4.29 & $0.96-19.18$ & 0.06 & 1.96 & $0.49-7.77$ & 0.34 \\
\hline CD8 cell count > 800 cells $/ \mu l$ & 0.86 & $0.21-3.55$ & 0.83 & 1.20 & $0.31-4.70$ & 0.79 \\
\hline CD4/CD8 ratio $>0.8$ & 1.68 & $0.40-6.96$ & 0.47 & 3.43 & $0.83-14.21$ & 0.09 \\
\hline Plasma HIV RNA viral load, <40 copies/ml & 7.00 & $0.64-76.7$ & 0.11 & 4.50 & $0.42-48.53$ & 0.21 \\
\hline Neutrophil count >3500cell/ $/ \mu \mathrm{l}$ & 0.57 & $0.14-2.38$ & 0.44 & 1.09 & $0.27-4.32$ & 0.90 \\
\hline CDC stage C & 0.47 & $0.08-2.83$ & 0.41 & 0.75 & $0.13-4.39$ & 0.75 \\
\hline Age $>50$ years & 1.44 & $0.35-5.95$ & 0.61 & 0.15 & $0.03-0.70$ & 0.02 \\
\hline Body mass index $\left(\mathrm{kg} / \mathrm{m}^{2}\right)$ & 4.54 & $0.79-25.97$ & 0.09 & 0.75 & $0.18-3.11$ & 0.69 \\
\hline CMV seropositivity & 1.33 & $0.19-9.46$ & 0.77 & 0.27 & $0.03-2.73$ & 0.27 \\
\hline Time since HIV infection & 0.60 & $0.14-2.59$ & 0.49 & 0.91 & $0.23-3.63$ & 0.90 \\
\hline History of bacterial pneumonia & 0.30 & $0.04-2.12$ & 0.23 & 0.47 & $0.07-3.26$ & 0.44 \\
\hline Nadir CD4 cell count <200 cells/ $\mu$ l & 0.52 & $0.12-2.31$ & 0.39 & 0.30 & $0.07-1.36$ & 0.12 \\
\hline Active tobacco use & 0.27 & $0.06-1.18$ & 0.08 & 0.87 & $0.22-3.52$ & 0.85 \\
\hline Prior PPV23 vaccine & 0.68 & $0.11-4.18$ & 0.68 & 0.43 & $0.07-2.62$ & 0.36 \\
\hline
\end{tabular}

PPV23, 23-valent pneumococcal polysaccharide vaccine.

Globally protected was defined as a patient who developed a protection for at least five of the tested serotypes targeted by PCV13.

Bold values indicate $p<0.05$.

TABLE 9 | Spearman's correlation coefficients between Elisa and OPA values at baseline, 1-, 6- and 12 month and for each serotype among 38 HIV-infected subjects with CD4 cell count $\geq 200$ cells/ $\mu$ l who received the PCV13 vaccine.

\begin{tabular}{|c|c|c|c|c|c|c|c|c|}
\hline \multirow[t]{2}{*}{ Serotype } & \multicolumn{2}{|c|}{ MO } & \multicolumn{2}{|c|}{ M1 } & \multicolumn{2}{|c|}{ M6 } & \multicolumn{2}{|c|}{ M12 } \\
\hline & Rho & $p$ & Rho & $p$ & Rho & $p$ & Rho & $\mathbf{p}$ \\
\hline 4 & 0.56 & 0.0003 & 0.46 & 0.003 & 0.54 & 0.0008 & 0.36 & 0.04 \\
\hline $6 B$ & 0.54 & 0.0005 & 0.42 & 0.009 & 0.42 & 0.01 & 0.42 & 0.01 \\
\hline $9 \mathrm{~V}$ & 0.44 & 0.006 & 0.38 & 0.02 & 0.50 & 0.002 & 0.48 & 0.004 \\
\hline 14 & 0.71 & $<0.0001$ & 0.72 & $<0.0001$ & 0.80 & $<0.0001$ & 0.81 & $<0.0001$ \\
\hline $18 \mathrm{C}$ & 0.52 & 0.0009 & 0.45 & 0.005 & 0.41 & 0.01 & 0.39 & 0.02 \\
\hline $19 F$ & 0.39 & 0.02 & 0.33 & 0.04 & 0.48 & 0.004 & 0.63 & $<0.0001$ \\
\hline $23 F$ & 0.46 & 0.004 & 0.65 & $<0.0001$ & 0.44 & 0.007 & 0.53 & 0.001 \\
\hline
\end{tabular}

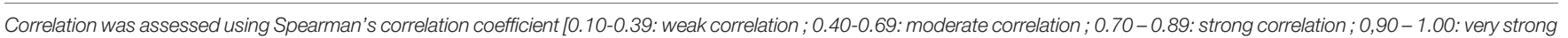
correlation (26)]. 
related questions in $\operatorname{PLHIV}(5,17,24,31,32)$ and immune response rate after a single dose of PCV13 have rarely been described. Most of the published studies concern a vaccination regimen of two doses of this conjugate vaccine whereas current guidelines for PLVIH recommend a single dose of PCV13 followed by PPV23 after at least 2 months, and even after a minimum of 1 year $(8,9)$.

We have observed in our study that most virologically suppressed and immunologically controlled PLHIV achieve adequate global immunological response and protection one month after a single PCV13 vaccination. According to ELISA and OPA measures, $57.9 \%$ and $63.2 \%$ were global responders and $92.1 \%$ and $78.9 \%$ were globally protected, respectively. Twelve months later, and without PPV23 subsequent vaccination, $64.7 \%$ and $55.9 \%$ of them were still globally protected against the tested serotypes, by ELISA and by OPA, respectively.

Only three studies have previously investigated vaccine response after a single dose of PCV13 in this population, either by ELISA or OPA, and the majority only analyzed two serotypes $(5,31,32)$. One study was performed in PLHIV stratified by CD4 T-cell count (less or more than 350 cells cells $/ \mu \mathrm{l}$ ), and reported serotype response rates using the same definition at one month assessed by OPA between $60.6 \%$ and $73.5 \%$ for the serotype $6 \mathrm{~B}$ and between $60.6 \%$ and $76.5 \%$ for the serotype $18 \mathrm{C}$ (5). We found comparable results by OPA analysis for these two serotypes. In a previous study using ELISA and OPA, the mean immunological response for the PCV13 vaccine was observed after one single dose of PCV13 for all but one (serotype 5 by ELISA and serotype 14 by OPA) of the 13 serotypes included in the PCV13 (32). Furthermore, the $1 \mu \mathrm{g} / \mathrm{ml}$ threshold was achieved for all serotypes but serotype 3. However, response rates were not provided. The increase in GMC and GMT between baseline and M1 were comparable with our study (for GMC: 2.44 to 5.40 -fold versus 2.4 to 3.45 -fold in our study respectively, and for GMT: 9.6 to 22.7 -fold versus 9.3 to 21.7 -fold in our study) (32). Similar results were reported in a third study stratifying PLHIV by CD4 cell count $>$ or $<400$ cells/ $\mu$, and analyzing immune response against the serotype 3 and 14 by ELISA (31).

Although disease prevention represents a better criterion than vaccine immune response for prophylactic vaccines like PCV13, we used pre-existing immunological criteria to define vaccineinduced response and protection against pneumococcal infection $(18,20,21)$.

In our study, we found that IgG antibody GMCs to six serotypes $(6 \mathrm{~B}, 9,14,19 \mathrm{~F}, 23 \mathrm{~F}$, and $7 \mathrm{~F})$ were already greater than or equal to $1 \mu \mathrm{g} / \mathrm{ml}$ before the PCV13 whereas GMT were never above the LLOQ for the seven serotypes tested. These results are consistent with those reported in a previous study for several serotypes (32). Moreover, half of our population was considered as already globally protected according to ELISA and only about $15 \%$ before PCV13 vaccination according to OPA, probably due to the presence of non-functional antibodies. This has already been observed in other studies (21). The patients with CD4 cell counts greater than 750 cells/ $\mu$ l were significantly more frequently protected at baseline according to ELISA analysis. Interestingly, we found no association with a previous PPV23 injection although most patients had already received one.
One month after PCV13 vaccination, we found that thirtyfive $(92.1 \%)$ and thirty (78.9\%) patients were globally protected according to ELISA and OPA measures, respectively. Only three studies have assessed vaccinal protection after a dose of PCV13 either in ELISA or OPA or both at one month after vaccination (5, 31, 32). Moreover, none of these studies reported global protection rates induced by PCV13 since their designs were different from our study. IgG levels targeting identical serotypes were comparable between our study and theirs. In a study, GMCs were similar with our study at one month and were above the $1 \mu \mathrm{g} / \mathrm{ml}$ threshold for all but one of 13 serotypes (serotype 5) (32). At one month, in this same study, OPA GMT was higher for serotypes $4,6 \mathrm{~B}, 9 \mathrm{~V}, 14$, and $18 \mathrm{C}$ but lower for serotypes $19 \mathrm{~F}$ and $23 \mathrm{~F}$ than in our study (32). In another study concerning only two serotypes (3 and 14), all patients were protected for serotype 14 one month after PCV13 vaccine but with a lower increase than in our study (31). In our study, at 1 month, the protection rate for each serotype ranged from $76.3 \%$ to $86.8 \%$ by OPA and $78.9 \%$ were globally protected. In a previous study, one-month immunological protection rate by OPA varied from $87.9 \%$ to $100 \%$ for serotype $6 \mathrm{~B}$ and from $75.8 \%$ to $94.1 \%$ for serotype $18 \mathrm{C}$ according to the CD4 cell count (5).

The durability of protection after PCV13 is another crucial point to further adapt vaccination strategy, and data concerning long-term protection after PCV13 in PLHIV are rare. At twelve months after PCV13 vaccine, we observed a decrease in the immunological global protection rate during follow-up and only $64.7 \%$ and $55.9 \%$ of the patients were still globally protected twelve months after their PCV13 vaccination, by ELISA and by OPA, respectively. To our knowledge, no such result has been published to date. Only one study assessed GMC one year after PCV13 vaccination for two serotypes (3 and 14), and for serotype 14 , which is known as an immunogenic serotype (33). We found higher antibody concentrations at M12 $(2.95 \mu \mathrm{g} / \mathrm{ml}$ vs. $7.6 \mu \mathrm{g} /$ $\mathrm{ml})$. Indeed, in our study, all patients were considered protected at M12 by ELISA for this serotype (70.6\% by OPA). There was also a progressive decrease in antibody concentration and opsonophagocytic titers over time for all serotypes.

There is also few one-year data with ELISA and OPA in patients with primary immune deficiency (21), multiple myeloma (18), or in elderly ( $>65$ years old) patients (34), which all observed a decrease of GMCs and GMTs over time. There is no data on the general population. In contrast to the CAPITA trial (34), which included patients over 65, we observed lower GMC values at M12 for all tested serotypes except for serotype 14 and 19F which were similar. By OPA, we observed lower GMT values at 12 months for all tested serotypes except for serotype $19 \mathrm{~F}$ and $23 \mathrm{~F}$ which were similar. However, no direct comparison was made.

We found a moderate correlation between antibody levels and opsonophagocytic titers at each time point for all serotypes, but a weak non-significant correlation for global protection at M12. Although part of this lack of association may be related to insufficient statistical power, and to the definitions of protection for both techniques $(35,36)$, these discrepancies may result from chronic viral infection-induced inflammation leading to the production of non-functional antibodies in PLHIV (37). 
Nevertheless, this poor correlation between ELISA and OPA results was reported in other studies in HIV-negative populations and may be related to functional variabilities between the antibodies produced. Comparison of affinity and/or avidity of antipneumococcal antibodies at different time points with higher and lower OPA titered sera would be a useful addition in the future to shed light on the discordance between ELISA and OPA results at different time points before and after vaccination. Consequently, in this population, as reported in elderly or in immunocompromised populations, GMC may represent insufficient surrogate markers to evaluate anti-pneumococcal immune protection $(13,37,38)$. At baseline, very few patients (15\%) were considered as globally protected when assessed by OPA before vaccination whereas most patients had antibody levels above $1 \mu \mathrm{g} / \mathrm{l}$ before vaccination by ELISA. Interestingly, we showed that one month after PCV13 vaccination, the GMC increased 2.4 to 3.45 -fold from baseline whereas GMT increase was higher, from 9.26 to 21.7-fold from baseline. Thus, PCV13 elicits the production of functional antibodies in this population and improves their opsonophagocytic ability. We found a significant correlation between ELISA and OPA for the global response with Rho $=0.67$ $(\mathrm{p}<0.0001)$, underlining once again the ability of the vaccine to stimulate the production of new functional antibodies by B cells.

Few articles have investigated the factors associated with PCV13 vaccine response. We have found that a CD4/CD8 ratio $>0.8$ was significantly associated with a better global response by OPA and that a CD4 nadir below 200 was significantly associated with a lower risk of global response by ELISA.

In previous studies, CD4 cell count seemed to represent the main factor associated with vaccine response, particularly with PPV23. Nadir CD4 count has previously been identified as a marker of poorer response $(5,37,39-42)$. CD4 cell count nadir strongly correlates with a lower level of memory B-cells despite CD4 cell count recovery. This could limit the physiological maintenance of the B-cell compartment, thereby limiting vaccine-induced response and protection (43). In a recent study, combination antiretroviral therapy at vaccination and virological control were correlated with a better response to PPV23 vaccination (42). The CD4/CD8 ratio correlates with chronic inflammation and immune senescence, even in virologically suppressed and immunologically controlled PLHIV and has been associated with poor hepatitis $\mathrm{A}$ and hepatitis $\mathrm{B}$ vaccine responses (44-46). This parameter also seems interesting for clinicians in relation to PCV13 vaccination, because subjects nowadays are mostly virologically suppressed patients, receiving HAART and with CD 4 cell count $>200$ cells/ $\mu$ l, even $>500$ cells $/ \mu \mathrm{l}$. One study suggested that the number or percentage of CD4 T lymphocytes co-expressing CD28 could predict responses to vaccination in PLHIV. This marker could be tested in future studies on pneumococcal vaccine responses (47).

In regard to factors associated with protection, we observed that people with a nadir CD4 cell count greater than 200 cells $/ \mu$ l and plasma HIV RNA viral load $<40$ copies $/ \mathrm{ml}$ at baseline were significantly more frequently globally protected one month after vaccination. In line with these results, Song et al. reported that the protection of PCV13 was significantly inferior among HIVinfected patients with CD4 T-cell count $<350$ cells/ $\mu \mathrm{L}$ compared to those with higher CD4 T-cell counts (5). Being a global responder at M1 was not significantly predictive of being globally protected at M12. PLHIV over 50 were less frequently globally protected at M12, which may be explained by immunosenescence promoted by aging and HIV. For HIV viral load, this has already been observed with antibody response or antibody specificity for various vaccines, including pneumococcal vaccines $(42,48,49)$, but there is no data to our knowledge for the association with protection with PCV13. This is consistent with most guidelines recommending vaccinating PLHIV who have a HIV viral load $<50$ copies/ml (50).

The strength of our study lies in the fact that it is the first to study the immunological efficacy of PCV13 up to one year in PLHIV, using ELISA and OPA with many serotypes. This allowed the assessment of global response and global protection and their associated factors in a population of immunologically controlled patients, which is the main type of population observed in developed countries today.

Our study also has also several limitations. The size of the sample limits the power to assess factors associated with immunological response and protection. The factors associated with global response or protection should be verified in other studies. The overrepresentation of PLVIH born in France may have introduced a selection bias, considering the potential variability of immune responses among PLVIH of different ancestry. Furthermore, all except three of the patients were men. In a previous study in adults aged over 50 years, men were found to have consistently higher levels of serotype-specific IgG than women in a study on PCV7 and/or PP23 responses. Nevertheless, the effect of sex on humoral responses to vaccines differs between different studies and the vaccines studied $(51,52)$. It should also be noted that the thresholds used in the present study and in others have not been validated in clinical studies focusing on pneumococcal pneumonia or invasive pneumococcal disease and with PLVIH.

In conclusion, our study demonstrated that a single PCV13 vaccination in a population of PLHIV (mostly immunovirologically controlled) led to immunological response and protection at one month for most patients, especially those with a CD4 nadir greater than 200 cells/ $\mu$ land a CD4/CD8 ratio greater than 0.8. In most patients PCV13 improved the opsonophagocytic activity of anti-pneumococcal antibodies. After six and twelve months without a boost vaccination by PPV23, the percentage of protected patients decreased but more than half of patients were still immunologically globally protected at one year. Considering discrepancies between ELISA and OPA results, OPA seems more appropriate in a research context to assess immunological protection in this population, using published thresholds.

\section{DATA AVAILABILITY STATEMENT}

The raw data supporting the conclusions of this article will be made available by the authors, without undue reservation. 


\section{ETHICS STATEMENT}

The studies involving human participants were reviewed and approved by Institutional review board of the Reims University Hospital. Written informed consent for participation was not required for this study in accordance with the national legislation and the institutional requirements.

\section{AUTHOR CONTRIBUTIONS}

AS, MH, JR, and AR designed the study. J-LB, DL, FB-S, and YN'G took care of patients. JR and GL collected

\section{REFERENCES}

1. Jauneikaite E, Tocheva AS, Jefferies JMC, Gladstone RA, Faust SN, Christodoulides M, et al. Current Methods for Capsular Typing of Streptococcus Pneumoniae. J Microbiol Methods (2015) 113:41-9. doi: 10.1016/j.mimet.2015.03.006

2. Siemieniuk RAC, Gregson DB, Gill MJ. The Persisting Burden of Invasive Pneumococcal Disease in HIV Patients: An Observational Cohort Study. BMC Infect Dis (2011) 11:314. doi: 10.1186/1471-2334-11-314

3. Cohen AL, Harrison LH, Farley MM, Reingold AL, Hadler J, Schaffner W, et al. Prevention of Invasive Pneumococcal Disease Among HIV-Infected Adults in the Era of Childhood Pneumococcal Immunization. AIDS (2010) 24:2253-62. doi: 10.1097/QAD.0b013e32833d46fd

4. Heffernan RT, Barrett NL, Gallagher KM, Hadler JL, Harrison LH, Reingold AL, et al. Declining Incidence of Invasive Streptococcus Pneumoniae Infections Among Persons With AIDS in an Era of Highly Active Antiretroviral Therapy, 1995-2000. J Infect Dis (2005) 191:2038-45. doi: 10.1086/430356

5. Song JY, Cheong HJ, Noh JY, Choi MJ, Yoon JG, Kim WJ. Immunogenicity and Safety of 13-Valent Pneumococcal Conjugate Vaccine in HIV-Infected Adults in the Era of Highly Active Antiretroviral Therapy: Analysis Stratified by CD4 T-Cell Count. Hum Vaccines Immunotherapeutics (2020) 16:169-75. doi: 10.1080/21645515.2019.1643677

6. Belmonti S, Rossetti B, Modica S, Paglicci L, Borghetti A, Ciccullo A, et al. Long-Term Serological Response to 13-Valent Pneumococcal Conjugate Vaccine Versus 23-Valent Polysaccharide Vaccine in HIV-Infected Adults. Infect Dis Ther (2019) 8:453-62. doi: 10.1007/s40121-019-0256-Z

7. Blanc A, Bonnet F, Brun-Vezinet F, Costagliola D, Dabis F, Delobel P, et al. Groupe Des Experts « Prise En Charge Médicale Des Personnes Infectées Par Le VIH ». (2018) 46.

8. Centers for Disease Control and Prevention (CDC). Use of 13-Valent Pneumococcal Conjugate Vaccine and 23-Valent Pneumococcal Polysaccharide Vaccine for Adults With Immunocompromising Conditions: Recommendations of the Advisory Committee on Immunization Practices (ACIP). MMWR Morb Mortal Wkly Rep (2012) 61:816-9.

9. Rubin LG, Levin MJ, Ljungman P, Davies EG, Avery R, Tomblyn M, et al. Executive Summary: 2013 IDSA Clinical Practice Guideline for Vaccination of the Immunocompromised Host. Clin Infect Dis (2014) 58:309-18. doi: $10.1093 / \mathrm{cid} / \mathrm{cit} 816$

10. Lazarus R, Clutterbuck E, Yu L-M, Bowman J, Bateman EA, Diggle L, et al. A Randomized Study Comparing Combined Pneumococcal Conjugate and Polysaccharide Vaccination Schedules in Adults. Clin Infect Dis (2011) 52:736-42. doi: 10.1093/cid/cir003

11. Rabian C, Tschöpe I, Lesprit P, Katlama C, Molina J-M, Meynard J-L, et al. ANRS 114 Pneumovac Study Group. Cellular CD4 T Cell Responses to the Diphtheria-Derived Carrier Protein of Conjugated Pneumococcal Vaccine and Antibody Response to Pneumococcal Vaccination in HIV-Infected Adults. Clin Infect Dis (2010) 50:1174-83. doi: 10.1086/651418

12. Burton RL, Nahm MH. Development and Validation of a Fourfold Multiplexed Opsonization Assay (MOPA4) for Pneumococcal Antibodies. Clin Vaccine Immunol (2006) 13:1004-9. doi: 10.1128/CVI.00112-06 the data. MB performed ELISA and OPA analyses. $\mathrm{MH}$ performed the statistical analysis. JR, $\mathrm{MH}$, and AS drafted the manuscript. AS supervised the study conduction. All authors contributed to data interpretation and analysis and revised the manuscript.

\section{ACKNOWLEDGMENTS}

We are indebted to Grace Stockton and Isabelle Kmiec for their precious help during the writing of this article.

13. Song JY, Moseley MA, Burton RL, Nahm MH. Pneumococcal Vaccine and Opsonic Pneumococcal Antibody. J Infect Chemother (2013) 19:412-25. doi: 10.1007/s10156-013-0601-1

14. French N, Moore M, Haikala R, Kayhty H, Gilks CF. A Case-Control Study to Investigate Serological Correlates of Clinical Failure of 23-Valent Pneumococcal Polysaccharide Vaccine in HIV-1-Infected Ugandan Adults. J Infect Dis (2004) 190:707-12. doi: 10.1086/421911

15. Chen M, Ssali F, Mulungi M, Awio P, Yoshimine H, Kuroki R, et al. Induction of Opsonophagocytic Killing Activity With Pneumococcal Conjugate Vaccine in Human Immunodeficiency Virus-Infected Ugandan Adults. Vaccine (2008) 26:4962-8. doi: 10.1016/j.vaccine.2008.06.093

16. Lesprit P, Pédrono G, Molina J-M, Goujard C, Girard P-M, Sarrazin N, et al. Immunological Efficacy of a Prime-Boost Pneumococcal Vaccination in HIVInfected Adults. AIDS (2007) 21:2425-34. doi: 10.1097/QAD.0b013e3282887e91

17. Garrido HMG, Schnyder JL, Tanck MWT, Vollaard A, Spijker R, Grobusch MP, et al. Immunogenicity of Pneumococcal Vaccination in HIV Infected Individuals: A Systematic Review and Meta-Analysis. EClinicalMedicine (2020) 29-30:100576. doi: 10.1016/j.eclinm.2020.100576

18. Bahuaud M, Bodilis H, Malphettes M, Maugard Landre A, Matondo C, Bouscary D, et al. Immunogenicity and Persistence of the 13-Valent Pneumococcal Conjugate Vaccine (PCV13) in Patients With Untreated Smoldering Multiple Myeloma (SMM): A Pilot Study. Heliyon (2017) 3: e00441. doi: 10.1016/j.heliyon.2017.e00441

19. Home. UAB Bacteriall Respiratory Pathogen Reference Laboratory. Available at: https://www.vaccine.uab.edu/ (Accessed September 5, 2021).

20. Lombardi F, Belmonti S, Fabbiani M, Morandi M, Rossetti B, Tordini G, et al. Immunogenicity and Safety of the 13-Valent Pneumococcal Conjugate Vaccine Versus the 23-Valent Polysaccharide Vaccine in Unvaccinated HIV-Infected Adults: A Pilot, Prospective Controlled Study. PLoS One (2016) 11:e0156523. doi: 10.1371/journal.pone.0156523

21. Robbins A, Bahuaud M, Hentzien M, Maestraggi Q, Barbe C, Giusti D, et al. The 13Valent Pneumococcal Conjugate Vaccine Elicits Serological Response and Lasting Protection in Selected Patients With Primary Humoral Immunodeficiency. Front Immunol (2021) 12:697128. doi: 10.3389/fimmu.2021.697128

22. Burton RL, Nahm MH. Development of a Fourfold Multiplexed Opsonophagocytosis Assay for Pneumococcal Antibodies Against Additional Serotypes and Discovery of Serological Subtypes in Streptococcus Pneumoniae Serotype 20. Clin Vaccine Immunol (2012) 19:835-41. doi: 10.1128/CVI.00086-12

23. Juergens C, Patterson S, Trammel J, Greenberg D, Givon-Lavi N, Cooper D, et al. Post Hoc Analysis of a Randomized Double-Blind Trial of the Correlation of Functional and Binding Antibody Responses Elicited by 13Valent and 7-Valent Pneumococcal Conjugate Vaccines and Association With Nasopharyngeal Colonization. Clin Vaccine Immunol (2014) 21:127781. doi: 10.1128/CVI.00172-14

24. Sadlier C, O’Dea S, Bennett K, Dunne J, Conlon N, Bergin C. Immunological Efficacy of Pneumococcal Vaccine Strategies in HIV-Infected Adults: A Randomized Clinical Trial. Sci Rep (2016) 6:32076. doi: 10.1038/srep32076

25. Tchalla EYI, Bhalla M, Wohlfert EA, Bou Ghanem EN. Neutrophils Are Required During Immunization With the Pneumococcal Conjugate Vaccine 
for Protective Antibody Responses and Host Defense Against Infection. J Infect Dis (2020) 222:1363-70. doi: 10.1093/infdis/jiaa242

26. Schober P, Boer C, Schwarte LA. Correlation Coefficients: Appropriate Use and Interpretation. Anesth Analg (2018) 126:1763-8. doi: 10.1213/ ANE.0000000000002864

27. Ho Y-L, Brandão AP, de Cunto Brandileone MC, Lopes MH. Immunogenicity and Safety of Pneumococcal Conjugate Polysaccharide and Free Polysaccharide Vaccines Alone or Combined in HIV-Infected Adults in Brazil. Vaccine (2013) 31:4047-53. doi: 10.1016/j.vaccine.2013.04.065

28. Peñaranda M, Payeras A, Cambra A, Mila J, Riera M. Conjugate and Polysaccharide Pneumococcal Vaccines Do Not Improve Initial Response of the Polysaccharide Vaccine in HIV-Infected Adults. AIDS (2010) 24:1226-8. doi: 10.1097/QAD.0b013e3283389de5

29. Feikin DR, Elie CM, Goetz MB, Lennox JL, Carlone GM, Romero-Steiner S, et al. Randomized Trial of the Quantitative and Functional Antibody Responses to a 7-Valent Pneumococcal Conjugate Vaccine and/or 23Valent Polysaccharide Vaccine Among HIV-Infected Adults. Vaccine (2001) 20:545-53. doi: 10.1016/S0264-410X(01)00347-4

30. Crum-Cianflone NF, Huppler Hullsiek K, Roediger M, Ganesan A, Patel S, Landrum ML, et al. A Randomized Clinical Trial Comparing Revaccination With Pneumococcal Conjugate Vaccine to Polysaccharide Vaccine Among HIV-Infected Adults. J Infect Dis (2010) 202:1114-25. doi: $10.1086 / 656147$

31. Farmaki PF, Chini MC, Mangafas NM, Tzanoudaki MT, Piperi CP, Lazanas MZ, et al. Immunogenicity and Immunological Memory Induced by the 13Valent Pneumococcal Conjugate Followed by the 23-Valent Polysaccharide Vaccine in HIV-Infected Adults. J Infect Dis (2018) 218:26-34. doi: 10.1093/ infdis/jiy 135

32. Glesby MJ, Watson W, Brinson C, Greenberg RN, Lalezari JP, Skiest D, et al. Immunogenicity and Safety of 13 -Valent Pneumococcal Conjugate Vaccine in HIV-Infected Adults Previously Vaccinated With Pneumococcal Polysaccharide Vaccine. J Infect Dis (2015) 212:18-27. doi: 10.1093/infdis/jiu631

33. Leggat DJ, Thompson RS, Khaskhely NM, Iyer AS, Westerink MAJ. The Immune Response to Pneumococcal Polysaccharides 14 and 23F Among Elderly Individuals Consists Predominantly of Switched Memory B Cells. J Infect Dis (2013) 208:101-8. doi: 10.1093/infdis/jit139

34. van Deursen AMM, van Houten MA, Webber C, Patton M, Scott DA, Patterson S, et al. Immunogenicity of the 13-Valent Pneumococcal Conjugate Vaccine in Older Adults With and Without Comorbidities in the Community-Acquired Pneumonia Immunization Trial in Adults (CAPiTA). Clin Infect Dis (2017) 65:787-95. doi: 10.1093/cid/cix419

35. Kamchaisatian W, Wanwatsuntikul W, Sleasman J, Tangsinmankong N. Validation of Current Joint American Academy of Allergy, Asthma \& Immunology and American College of Allergy, Asthma and Immunology Guidelines for Antibody Response to the 23-Valent Pneumococcal Vaccine Using a Population of HIV-Infected Children. J Allergy Clin Immunol (2006) 118:1336-41. doi: 10.1016/j.jaci.2006.09.036

36. Orange JS, Ballow M, Stiehm ER, Ballas ZK, Chinen J, de la Morena M, et al. Use and Interpretation of Diagnostic Vaccination in Primary Immunodeficiency: A Working Group Report of the Basic and Clinical Immunology Interest Section of the American Academy of Allergy, Asthma \& Immunology. J Allergy Clin Immunol (2012) 130:S1-S24. doi: 10.1016/j.jaci.2012.07.002

37. Leggat DJ. Response to Pneumococcal Polysaccharide Vaccination in Newly Diagnosed HIV-Positive Individuals. J AIDS Clin Res (2015) 6(2):419. doi: 10.4172/2155-6113.1000419

38. Romero-Steiner S, Frasch CE, Carlone G, Fleck RA, Goldblatt D, Nahm MH. Use of Opsonophagocytosis for Serological Evaluation of Pneumococcal Vaccines. Clin Vaccine Immunol (2006) 13:165-9. doi: 10.1128/CVI.13. 2.165-169.2006

39. Hung C-C, Chang S-Y, Su C-T, Chen Y-Y, Chang S-F, Yang C-Y, et al. A 5Year Longitudinal Follow-Up Study of Serological Responses to 23-Valent Pneumococcal Polysaccharide Vaccination Among Patients With HIV Infection Who Received Highly Active Antiretroviral Therapy*: Pneumococcal Vaccination and HAART. HIV Med (2010) 11:54-63. doi: $10.1111 / j .1468-1293.2009 .00744 . x$

40. Lu C-L, Hung C-C, Chuang Y-C, Liu W-C, Su C-T, Hsiao C-F, et al. Comparison of Serologic Responses to Vaccination With One Dose or
Two Doses of 7-Valent Pneumococcal Conjugate Vaccine in HIV-Infected Adult Patients. Vaccine (2012) 30:3526-33. doi: 10.1016/j.vaccine. 2012.03.070

41. Rodriguez-Barradas MC, Alexandraki I, Nazir T, Foltzer M, Musher DM, Brown S, et al. Response of Human Immunodeficiency Virus-Infected Patients Receiving Highly Active Antiretroviral Therapy to Vaccination With 23-Valent Pneumococcal Polysaccharide Vaccine. Clin Infect Dis (2003) 37:438-47. doi: 10.1086/375841

42. Tsachouridou O, Christaki E, Skoura L, Georgiou A, Nanoudis S, Vasdeki D, et al. Predictors of Humoral Response to Recommended Vaccines in HIVInfected Adults. Comp Immunol Microbiol Infect Dis (2017) 54:27-33. doi: 10.1016/j.cimid.2017.07.006

43. Pensieroso S, Galli L, Nozza S, Ruffin N, Castagna A, Tambussi G, et al. B-Cell Subset Alterations and Correlated Factors in HIV-1 Infection. AIDS (2013) 27:1209-17. doi: 10.1097/QAD.0b013e32835edc47

44. Fritzsche C, Bergmann L, Loebermann M, Glass A, Reisinger EC. Immune Response to Hepatitis A Vaccine in Patients With HIV. Vaccine (2019) 37:2278-83. doi: 10.1016/j.vaccine.2019.02.064

45. Fuster F, Vargas JI, Jensen D, Sarmiento V, Acuña P, Peirano F, et al. CD4/ CD8 Ratio as a Predictor of the Response to HBV Vaccination in HIVPositive Patients: A Prospective Cohort Study. Vaccine (2016) 34:1889-95. doi: 10.1016/j.vaccine.2016.02.055

46. Noël L, Tubiana R, Simon A, Valantin M-A, Palich R, Blanc C, et al. Low Immune Response Rate of HIV-Infected Patients to a Single Injection of Hepatitis A Vaccine. Infect Dis Now (2021) 51:94-6. doi: 10.1016/ j.medmal.2020.09.016

47. Lange CG, Lederman MM, Medvik K, Asaad R, Wild M, Kalayjian R, et al. Nadir CD4+ T-Cell Count and Numbers of CD28+ CD4+ T-Cells Predict Functional Responses to Immunizations in Chronic HIV-1 Infection. AIDS (2003) 17:2015-23. doi: 10.1097/00002030-200309260-00002

48. Barte H, Horvath TH, Rutherford GW. Yellow Fever Vaccine for Patients With HIV Infection. Cochrane Database Syst Rev (2014) 23(1):CD010929. doi: 10.1002/14651858.CD010929.pub2

49. Lu C-L, Chang S-Y, Sun H-Y, Liu W-C, Tseng Y-T, Hsieh C-Y, et al. Impact of Vaccination With Seven-Valent Pneumococcal Conjugate Vaccine on Virologic and Immunologic Outcomes Among HIV-Infected Adult Patients in the Era of Highly Active Antiretroviral Therapy. J Formos Med Assoc (2012) 111:445-51. doi: 10.1016/j.jma.2011.06.027

50. Vaccination. EACS Guidelines. Available at: https://eacs.sanfordguide.com/ prevention-non-infectious-co-morbidities/vaccination (Accessed November 11, 2021).

51. Goldblatt D, Southern J, Andrews N, Ashton L, Burbidge P, Woodgate S, et al. The Immunogenicity of 7-Valent Pneumococcal Conjugate Vaccine Versus 23-Valent Polysaccharide Vaccine in Adults Aged 50-80 Years. Clin Infect Dis (2009) 49:1318-25. doi: 10.1086/606046

52. Gubbels Bupp MR, Potluri T, Fink AL, Klein SL. The Confluence of Sex Hormones and Aging on Immunity. Front Immunol (2018) 9:1269. doi: 10.3389 /fimmu.2018.01269

Conflict of Interest: The authors declare that the research was conducted in the absence of any commercial or financial relationships that could be construed as a potential conflict of interest.

Publisher's Note: All claims expressed in this article are solely those of the authors and do not necessarily represent those of their affiliated organizations, or those of the publisher, the editors and the reviewers. Any product that may be evaluated in this article, or claim that may be made by its manufacturer, is not guaranteed or endorsed by the publisher.

Copyright (c) 2021 Romaru, Bahuaud, Lejeune, Hentzien, Berger, Robbins, Lebrun, N'Guyen, Bani-Sadr, Batteux and Servettaz. This is an open-access article distributed under the terms of the Creative Commons Attribution License (CC BY). The use, distribution or reproduction in other forums is permitted, provided the original author(s) and the copyright owner(s) are credited and that the original publication in this journal is cited, in accordance with accepted academic practice. No use, distribution or reproduction is permitted which does not comply with these terms. 